\title{
Análise da produção científica de área de conhecimento específico: caracterização do tema requisitos do cliente do mercado da construção civil
}

\author{
Analysis of the academic output in a specific field of \\ knowledge: characterization of the theme client \\ requirements in the building market
}

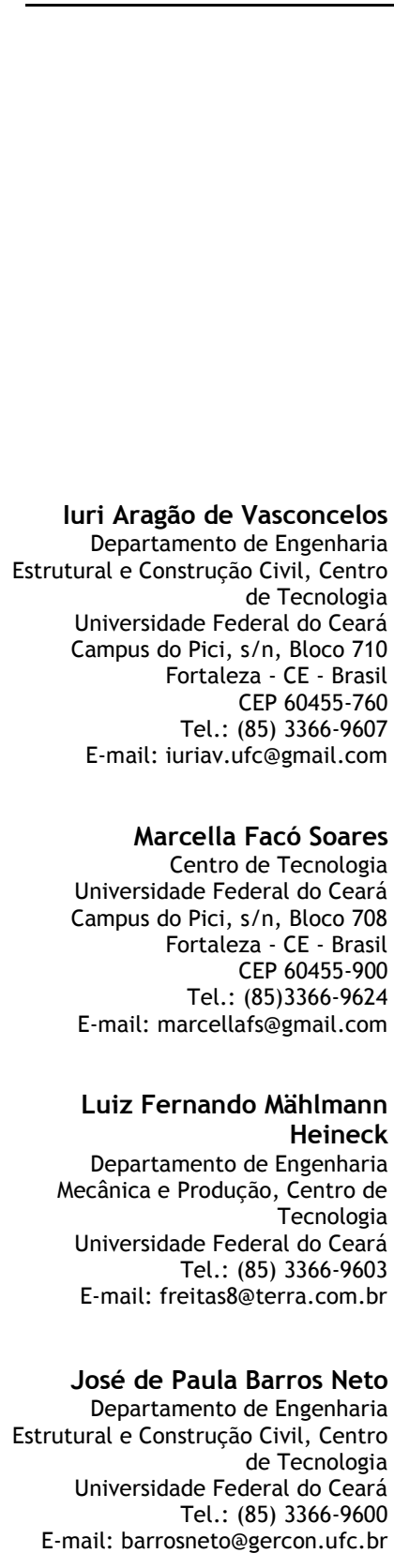

Recebido em 02/02/12 Aceito em 09/02/13

\section{luri Aragão de Vasconcelos \\ Marcella Facó Soares \\ Luiz Fernando Mählmann Heineck José de Paula Barros Neto}

evolução que vem ocorrendo nas últimas décadas no processo de gerenciar projetos de construção tem apontado fortemente para a priorização dos interesses dos clientes. Entretanto, na comunidade acadêmica, o entendimento de como ocorrem as pesquisas relacionadas a este tema ainda é precário, necessitando de uma melhor compreensão das tendências existentes e diretrizes para estudos posteriores. Este artigo tem por objetivo a caracterização da produção científica brasileira sobre requisitos dos clientes do mercado da construção civil, um dos temas de pesquisa que têm mais crescido na área de Tecnologia do Ambiente Construído. Foi realizado uma análise de 124 artigos relativos ao tema publicados no Brasil entre 2005 a 2011. Este estudo foi focado em três eventos, SIBRAGEC, ENTAC e IGLC, além da Revista Ambiente Construído. Entretanto, alguns artigos provenientes de outros eventos também foram considerados, visando a ampliar a discussão. Foram apontadas as linhas de pesquisas mais abordadas, as universidades e autores que mais publicaram, bem como a titulação destes. Foram identificadas as estratégias de pesquisa utilizadas nos artigos, assim como quantificadas as referências mais citadas e os tipos de bibliografias mais mencionados.

Palavras-chave: Requisitos dos clientes. Construção de edificações. Produção científica. Análise bibliométrica.

\section{Abstract}

The evolution that has occurred in recent decades in the process of managing construction projects points out strongly for the prioritization of clients' interests. However, in the academic community, the understanding about how research is carried out in this field is still poor, and there is also a need to identify existing trends as well as to directions for future studies. This paper aims to characterize the Brazilian scientific production on client requirements in the building market, which is one of the fast growing fields in the area of Built Environment. This article was based on the analysis of 124 papers published between 2005 and 2011. This study was focused on three conferences, SIBRAGEC, ENTAC and IGLC, as well as on Ambiente Construido journal. Moreover, the study included some papers from other events with the aim of extending the discussion. The most popular research topics were identified, as well as the universities and authors with the largest number of publications and also their academic qualifications. The research strategies used in the papers were pointed out, and the most cited references and types of citations were quantified.

Keywords: Client requirement. Building construction. Academic output. Bibliometric analysis. 


\section{Introdução}

A atual situação econômica brasileira favorece o crescimento que vem ocorrendo nos últimos anos no meio científico e tecnológico do país. Contudo, ainda há carência em logística e recursos aplicados no desenvolvimento de pesquisas. Uma maneira de diminuir essa problemática é realizar estudos que organizem a produção científica de áreas do conhecimento, o que pode fornecer diretrizes para um melhor investimento em processo de linhas de pesquisas (PITHAN et al., 2005).

Uma das ferramentas mais utilizadas para realizar estudos de levantamento e análise de produção científica é a abordagem bibliométrica. Fonseca (1986) define a bibliometria como uma técnica quantitativa e estatística de medição de parâmetros de produção e dispersão do conhecimento.

Para Macias-Chapula (1998), a bibliometria possibilita realizar estudos comparativos de como a produção científica se desenvolveu até o momento da análise, permitindo a prospecção e a tomada de decisão quanto aos novos investimentos a serem aplicados na área de conhecimento enviesada.

Segundo Rousseau (1998), a realização de uma análise bibliométrica possibilita uma visualização mais eficiente do processo de pesquisa, o que garante melhor qualidade de resultados $\mathrm{e}$ diretrizes.

Tendo em vista a escassez de estudos brasileiros voltados ao mapeamento da produção científica referente ao tema de requisitos do cliente, faz-se necessário preencher essa lacuna do conhecimento. Além disso, o ato de realizar uma apuração e análise da atividade científica de determinado assunto possibilita o direcionamento do enfoque dado a ela, reduzindo recursos empregados e aumentando a qualidade da evolução das pesquisas (SAES, 2000).

O tema de pesquisa voltado para a abordagem dos Requisitos dos Clientes (ou Usuários, que nesta pesquisa são tomados como iguais) da construção civil começa a ganhar espaço devido às novas tendências de mercado, em que os clientes passam a interagir e compreender mais sobre o ambiente construído e sobre o valor agregado a ele.

Pesquisadores nacionais e internacionais estão entrando nesse aspecto da gestão de projetos devido à crescente importância dos consumidores do ramo imobiliário em vista da evolução desse setor industrial. Vários autores apontam a relevância das necessidades dos clientes no processo de desenvolvimento de projetos.
Koskela (2000), por exemplo, afirma que os princípios tradicionais de gestão de obras visam apenas à entrega do produto, enquanto as novas filosofias gerenciais buscam a entrega do produto seguida da satisfação do cliente, redução dos gastos e perdas, geração de valor para os consumidores e valorização da credibilidade das empresas.

Quando as especificações de um produto não são atendidas após a execução de um processo e existe a necessidade de retrabalho, significa que atividades de processamento foram executadas sem agregar valor (FORMOSO, 2002). Uma estrutura de trabalho bem definida, com aspectos projetuais e executivos alinhados, bem como a inserção das necessidades dos clientes na cadeia produtiva (projeto e produção), garantindo o fluxo de informações e suprimentos, possibilita a agregação de valor ao produto, empresa e consumidor (BALLARD, 2000). Portanto, levar em consideração as necessidades dos clientes, na fase de projetos e na etapa construtiva, é fundamental para enxugar o processo de desenvolvimento do produto.

Assim, este trabalho tem como objetivo a caracterização da produção científica relativa ao tema de requisitos dos clientes do mercado da construção civil, dando ênfase aos eventos Simpósio Brasileiro em Gestão e Economia da Construção (SIBRAGEC), Encontro Nacional em Tecnologia do Ambiente Construído (ENTAC), ambos nacionais, e International Group for Lean Construction (IGLC), internacional, bem como a Revista Ambiente Construído, pois são entendidos como eventos de destaque na área de gestão da construção. Outros congressos foram pesquisados, dos quais se extraiu uma quantidade menor de artigos, pois o foco do trabalho foi dado aos eventos supracitados, com o intuito de fomentar a discussão proposta neste artigo. Todos esses eventos e periódico apresentam comissão científica que avalia e seleciona criteriosamente os trabalhos submetidos a eles.

Este estudo, que aborda o período entre 2005 e 2011, apresenta a dispersão do número de artigos ao longo do período, as linhas de pesquisas mais abordadas, as universidades e autores que mais publicaram, a titulação destes, as estratégias de pesquisa utilizadas nos artigos, as referências mais citadas e os tipos de bibliografias mais utilizados.

Este trabalho possibilita, então, uma maior compreensão da evolução das pesquisas voltadas ao tema de requisitos dos clientes do mercado da construção civil como área do conhecimento 
pertinente à Tecnologia do Ambiente Construído, contribuindo com o preenchimento dessa lacuna de conhecimento e, conforme os autores citados acima, favorecendo o encaminhamento de futuras pesquisas mais bem direcionadas e focadas.

\section{Revisão bibliográfica}

\section{Requisitos dos clientes}

Gerenciamento de requisitos dos clientes é entendido como a identificação e compreensão deles, analisando suas necessidades e expectativas, além do processamento da informação, a fim de tornar essas informações acessíveis e de fácil entendimento aos interessados no processo de gestão de projeto (MIRON; FORMOSO, 2003).

Para iniciar um projeto, o processo de coleta de dados quanto às necessidades do cliente é entendido como o embasamento prévio que instrui os projetistas e construtores na concepção e execução do empreendimento. Normalmente, são documentados os requisitos relativos à funcionalidade, custo, prazo, padrão de acabamento, conforto ambiental e modificações no leiaute das unidades (FORMOSO; LEITE; MIRON, 2011; KAMARA; ANUMBA; EVBUOMWAN, 2002).

Para Barret, Hudson e Stanley (1999), a informação gerada nesse processo de coleta de requisitos dos clientes deve ser utilizada não somente na etapa de pré-projeto, mas também durante todo o seu ciclo de vida (concepção, construção e pós-ocupação), de modo que as necessidades dos clientes possam ser mais bem compreendidas, adaptadas e aplicadas. Contudo, a aplicação desses procedimentos ainda é precária na realidade das empresas, que, muitas vezes, não fazem uso de nenhuma forma de coleta de dados e se baseiam em suas próprias experiências.

Segundo Bruce e Cooper (2000), outras indústrias têm utilizado o processo de coleta de requisitos tanto com clientes externos quanto internos, de modo a otimizar a distribuição de recursos, favorecer condições de competitividade mercadológica e potencializar o crescimento tecnológico.

Com esses conceitos em mente, a necessidade em desenvolver estudos focados e alta qualidade na área de requisitos dos clientes é um marco real na comunidade científica e empresarial, especialmente quando diversos autores apontam que o desenvolvimento de projetos no mercado da construção tem-se tornado cada vez mais complexo, o que sugere a importância em investimentos na investigação e gestão dos requisitos dos clientes (BAILETTI; LITVA, 1995;
BARRETT; HUDSON; STANLEY, 1999; HUOVILA; SERÉN, 1998; KAMARA; ANUMBA; EVBUOMWAN, 2002; KOSKELA, 2000; SHEN et al., 2004).

\section{Pesquisa bibliométrica}

Como anteriormente explicitado, a bibliometria consiste na abordagem quantitativa de análise comparativa de conteúdos (produção científica). Além do estudo bibliométrico, outras técnicas bastante empregadas nesse intuito são a cienciometria e a informetria, que podem ser interpretadas como ramificações da bibliometria (TAGUE-SUTCLIFFE, 1992).

Comparativamente, a bibliometria, a cienciometria e a informetria são equivalentes com relação ao tipo de levantamento realizado (quantitativo), contudo divergem com relação ao objeto estudado, as variáveis coletadas, os métodos específicos de análise e a finalidade do estudo (TAGUESUTCLIFFE, 1992).

Diversos autores apontam para o crescimento na utilização de indicadores bibliométricos como embasamento do conhecimento da produção científica, de modo a nortear o planejamento de execução de pesquisas (GUEDES; BORSCHIVER, 2005; KRZYZANOWSKI; FERREIRA, 1998; MUGNANI; JANNUZZI; QUONIAM, 2004). Dessa forma, os estudos bibliométricos caracterizam a produção científica e fornecem indicações de como prosseguir novos estudos (MACIAS-CHAPULA, 1998).

\section{Método de pesquisa}

Para a realização deste levantamento, fez-se uma seleção prévia de artigos através de rotinas de busca (acervos da Revista Ambiente Construído e do IGLC; e Infohab), fazendo-se uso das palavraschave descritas abaixo. Observou-se nessa préseleção uma relevância do periódico da revista Ambiente Construído e dos eventos SIBRAGEC, ENTAC e IGLC, sendo os três primeiros nacionais e o último internacional, nos quais se buscou, posteriormente, o levantamento através dos anais relacionados ao período em análise. Outros eventos (observados na seção 4) foram adicionados à análise, fazendo-se uso somente dos dados obtidos com as rotinas de busca.

Selecionaram-se os artigos publicados no período de 2005 a 2011 por duas razões: para evitar dados defasados; e por ser um intervalo entendido como adequado para representar as características da temática estudada, dado que a partir do projeto de doutorado de Miron (2005) houve a sistematização do conteúdo sobre requisito de cliente. Vale 
ressaltar que, apesar de existirem trabalhos anteriores ao período de 2004 , inclusive da própria autora, os mesmos são estudos iniciais, sem uma sistemática objetiva sobre o assunto. Já a tese de Miron (2005) unificou e sistematizou o tema.

Como critério de pré-seleção, analisaram-se os artigos a partir das seguintes palavras-chave, procuradas nos títulos, resumos e corpos dos artigos: Requisitos, Satisfação e Reclamação (em interação com) Cliente, Usuário ou Consumidor; PDP - Processo de Desenvolvimento de Produto; Geração de Valor (com visão no cliente); e Percepção de Valor. A busca foi feita também para os termos traduzidos para o inglês, perfazendo: Requirement; Satisfaction e Complaint interagindo com Client, User ou Consumer; Product Development; Value; e Perceived Value. Esse conjunto de termos foi definido por apresentar relevância no que tange ao contexto analisado.

Entre os artigos pré-selecionados, elegeram-se aqueles de diversas linhas de pesquisa, conforme descritas mais à frente nesta seção, que abordavam requisitos dos clientes, fazendo-se uma análise deles, o que resultou na escolha de 124 artigos. Esse exame foi feito pelos autores de forma qualitativa, observando-se o título, as palavraschave, o resumo, os objetivos, a metodologia e as conclusões.

Para a escolha dos dados a serem avaliados, buscaram-se informações que pudessem ser relevantes à evolução da pesquisa científica no estudo do requisito do cliente. Respeitaram-se ainda os limites da bibliometria como um estudo quantitativo, como dito anteriormente e conforme indica a afirmativa abaixo no que diz respeito ao desígnio dos indicadores bibliométricos:

\section{[...] a questão que se impõe é para encontrar métodos e técnicas que permitam extrair sentido nessa massa de informação, sem, no entanto, ter que ler documento por documento, o que tornaria inviável o processo. (MACEDO DOS SANTOS, 2003 ${ }^{1}$ apud ROUSEAU-HANS; THIL, 1997).}

Portanto, iniciou-se a etapa de caracterização da produção científica coletando-se dados sobre os autores, instituições de vínculo, métodos de pesquisa descritos nos artigos e aos respectivos temas. Foram também coletados dados referentes às citações, incluindo tipo de bibliografia e os trabalhos mais citados. Para algumas das variáveis levantadas foram realizadas análises temporais, de

${ }^{1}$ MACEDO DOS SANTOS, Raimundo Nonato. Produção Científica: por que medir? O que medir? Revista Digital de Biblioteconomia e Ciência da Informação, Campinas, v. 1, n. 1, p. 22 38, jul./dez. 2003. forma a caracterizar algumas tendências ao longo do tempo. A seguir, são resumidamente descritos os dados coletados:

(a) ano de publicação: relativo ao ano de publicação dos artigos. Serve para realizar análises temporais e de dispersão dos trabalhos;

(b) nomes dos autores: tentou-se usar a forma mais completa do nome do autor indicado na publicação, sendo somente em alguns casos mantida abreviação (Dados não expostos);

(c) titulação acadêmica do autor: manteve-se a indicação do autor na publicação. Caso a titulação acadêmica não fosse fornecida diretamente nos artigos, buscou-se essa informação em outros artigos publicados pelo mesmo autor na mesma edição do evento ou em outros eventos do mesmo ano. Nos casos cuja informação não pôde ser levantada, enquadrou-se como "não indicado";

(d) vínculo institucional do autor: utilizou-se o mesmo procedimento que para titulação;

(e) estratégia de pesquisa: verificou-se nos corpos dos artigos a classificação dada pelos autores quanto a sua estratégia de pesquisa. A leitura se deu inicialmente pela seção correspondente à estratégia, depois resumo, introdução, resultados e conclusão, nesta ordem. A classificação encontrada foi: estudo teórico, estudo de caso e levantamento. Para aqueles que usaram uma metodologia nova, que não se encaixava nos citados, foram classificados como "Aplicação de Metodologia". Alguns, entretanto, não enquadraram sua metodologia em nenhum tipo, então o artigo foi caracterizado como tendo metodologia "não indicada".;

(f) detalhamento ou não do método de pesquisa: teve-se como objetivo identificar se haviam sido detalhadas no artigo as etapas e as principais ferramentas de coleta de dados. Esse dado foi obtido através da leitura técnica de partes do artigo: seção correspondente à estratégia, resumo, introdução e resultados, consecutivamente;

(g) linhas de pesquisa: foram estabelecidas a partir das palavras-chave, de uma análise do resumo e das principais temáticas que cercam o assunto. Sendo assim, os artigos foram divididos em quatro grupos: Geração de Valor, Gestão de Projeto, Gestão de Requisitos e Avaliação de PósOcupação (APO). A definição desta classificação ocorreu subjetivamente, visto que ainda não existe uma segmentação clara em temas consolidados nessa subárea. Além disso, alguns artigos foram classificados dentro de um ou mais temas devido à própria natureza sistêmica do conhecimento envolvido; 
(h) tipo de bibliografia: as referências bibliográficas dos artigos foram classificadas em: artigo de periódico, livro, tese e dissertações, artigo de evento, relatório de pesquisa, norma técnica, boletim técnico, legislação e "outros". Nesta última categoria foram considerados artigos não publicados, notas de aula, artigos de revista, textos para discussão, apostilas de caráter didático e os demais tipos de obras que não se enquadram na classificação proposta. Os dados supracitados foram coletados por meio do levantamento das referências bibliográficas em todos os artigos da amostra; e

(i) referências bibliográficas mais citadas: a identificação se deu a partir de um levantamento e análise entre as referências bibliográficas dos trabalhos estudados.

\section{Resultados da pesquisa}

\section{Análise da distribuição das publicações ao longo dos anos}

Devido a sua importância dentro do gerenciamento, o tema "requisitos do cliente do mercado da construção civil" mantém-se sempre presente nos eventos, porém sua frequência tem variado ao longo dos sete anos analisados. Na Tabela 1, observa-se uma queda na quantidade de artigos publicados a partir de 2008, que pode ser atribuída a um possível foco em outros temas na área de gerenciamento em construção.

Os anos mais significativos quanto à publicação dos artigos levantados foram 2005, 2006 e 2008, todos com mais de 20 artigos, enquanto o ano com menos publicações foi 2007 , com somente 6 . O evento que apresentou maior número de publicações foi o ENTAC, que não ocorre em anos ímpares, como em 2007.

Pela Tabela 2, percebe-se uma coerência entre os números totais da Tabela 1, ratificando seus dados. O mínimo de autores que abordaram o tema em um mesmo ano é de 22, e o máximo, de 74 (aproximadamente 3,5 vezes maior). Observa-se ainda que o SIBRAGEC e o ENTAC apresentaram publicações em todos os anos que ocorreram.

A Tabela 3 apresenta uma interessante curva de crescimento e decaimento das publicações relacionando os eventos, o número de universidades envolvidas e o período. Comparando-a com a Tabela 1, percebe-se que há artigos que incluem a participação de mais de uma universidade. Essa tabela apresenta convergência em relação às anteriores, com seus máximos e mínimos nos mesmos anos já citados, inclusive em cada evento individualmente.

\section{Análise das publicações por linha de pesquisa}

Analisando-se as linhas de pesquisa e sua distribuição de frequência ao longo dos anos pela Tabela 4, a maior parte dos artigos foi sobre Gestão de Requisitos, sendo este também o que apresentou maior desvio padrão ao longo do período analisado. A linha de pesquisa que apresentou menor desvio padrão foi Geração de Valor. É importante ratificar, porém, que esses dados não representam as linhas de pesquisa nos eventos de forma geral, somente quanto à abordagem de requisitos do cliente. Representa também o quanto os requisitos dos clientes estão diretamente relacionados com a geração de valor, partindo do princípio de que o valor é baseado principalmente na percepção do cliente.

\section{Tabela 1 - Evento x Ano x Número de Artigos}

\begin{tabular}{|c|c|c|c|c|c|c|c|c|c|c|c|}
\hline \multirow{2}{*}{ Período } & \multicolumn{6}{|c|}{ Eventos Nacionais } & \multicolumn{3}{|c|}{ Eventos Internacionais } & \multirow{2}{*}{$\begin{array}{c}\text { Revista } \\
\text { AC }\end{array}$} & \multirow{2}{*}{$\begin{array}{l}\text { Total } \\
\text { p/Ano }\end{array}$} \\
\hline & ENTECA & SMHIS & SIBRAGEC & ENTAC & ENCAC & SBQP $^{2}$ & $\mathbf{P B S}^{2}$ & PLEA $^{3}$ & IGLC & & \\
\hline 2005 & 1 & 2 & 14 & 0 & 0 & 0 & 0 & 0 & 4 & 3 & 24 \\
\hline 2006 & 0 & 0 & 0 & 20 & 0 & 0 & 0 & 0 & 4 & 2 & 26 \\
\hline 2007 & 0 & 0 & 3 & 0 & 0 & 0 & 1 & 0 & 2 & 0 & 6 \\
\hline 2008 & 0 & 0 & 0 & 16 & 0 & 0 & 0 & 1 & 5 & 0 & 22 \\
\hline 2009 & 0 & 0 & 5 & 0 & 2 & 7 & 0 & 0 & 2 & 3 & 19 \\
\hline 2010 & 0 & 0 & 0 & 8 & 0 & 0 & 0 & 0 & 5 & 0 & 13 \\
\hline 2011 & 0 & 0 & 1 & 0 & 0 & 0 & 0 & 0 & 9 & 4 & 14 \\
\hline $\begin{array}{l}\text { Total p/ } \\
\text { Evento }\end{array}$ & 1 & 2 & 23 & 44 & 2 & 7 & 1 & 1 & 31 & 12 & 124 \\
\hline
\end{tabular}

\footnotetext{
2 Deve-se ratificar que o SBQP foi criado somente em 2009, o que justifica a ausência de publicações anteriores a esse ano.

2 PBS: Proceedings of Building Simulation.

${ }^{3}$ PLEA: Conference on Passive and Low Energy Architecture.
} 
Tabela 2 - Evento x Ano x Número de Autores

\begin{tabular}{|c|c|c|c|c|c|c|c|c|c|c|c|}
\hline \multirow{2}{*}{ Período } & \multicolumn{6}{|c|}{ Eventos Nacionais } & \multicolumn{3}{|c|}{ Eventos Internacionais } & \multirow{2}{*}{$\begin{array}{c}\text { Revista } \\
\text { AC }\end{array}$} & \multirow{2}{*}{$\begin{array}{c}\text { Total } \\
\text { p/ } \\
\text { Ano }\end{array}$} \\
\hline & ENTECA & SMHIS & SIBRAGEC & ENTAC & ENCAC & SBQP & PBS & PLEA & IGLC & & \\
\hline 2005 & 3 & 3 & 45 & - & 0 & - & 0 & 0 & 10 & 6 & 67 \\
\hline 2006 & 0 & 0 & - & 62 & 0 & - & 0 & 0 & 6 & 6 & 74 \\
\hline 2007 & 0 & 0 & 12 & - & 0 & - & 4 & 0 & 6 & 0 & 22 \\
\hline 2008 & 0 & 0 & - & 44 & 0 & - & 0 & 2 & 13 & 0 & 59 \\
\hline 2009 & 0 & 0 & 19 & - & 5 & 17 & 0 & 0 & 7 & 14 & 62 \\
\hline 2010 & 0 & 0 & - & 26 & 0 & 0 & 0 & 0 & 17 & 0 & 43 \\
\hline 2011 & 0 & 0 & 3 & - & 0 & 0 & 0 & 0 & 27 & 13 & 43 \\
\hline $\begin{array}{l}\text { Total p/ } \\
\text { Evento }\end{array}$ & 3 & 3 & 79 & 132 & 5 & 17 & 4 & 2 & 86 & 39 & 327 \\
\hline
\end{tabular}

Tabela 3 - Evento x Ano x Número de Universidades

\begin{tabular}{|c|c|c|c|c|c|c|c|c|c|c|c|}
\hline \multirow[b]{2}{*}{ Período } & \multicolumn{6}{|c|}{ Eventos Nacionais } & \multicolumn{3}{|c|}{ Eventos Internacionais } & \multirow{2}{*}{$\begin{array}{c}\text { Revista } \\
\text { AC }\end{array}$} & \multirow{2}{*}{$\begin{array}{c}\text { Tota } \\
\text { p/ } \\
\text { Ano }\end{array}$} \\
\hline & ENTECA & SMHIS & SIBRAGEC & ENTAC & ENCAC & SBQP & PBS & PLEA & IGLC & & \\
\hline 2005 & 3 & 1 & 14 & 0 & 0 & 0 & 0 & 0 & 3 & 4 & 25 \\
\hline 2006 & 0 & 0 & 0 & 14 & 0 & 0 & 0 & 0 & 2 & 2 & 18 \\
\hline 2007 & 0 & 0 & 2 & 0 & 0 & 0 & 1 & 0 & 2 & 0 & 5 \\
\hline 2008 & 0 & 0 & 0 & 8 & 0 & 0 & 0 & 1 & 6 & 0 & 15 \\
\hline 2009 & 0 & 0 & 5 & 0 & 2 & 6 & 0 & 0 & 3 & 3 & 19 \\
\hline 2010 & 0 & 0 & 0 & 8 & 0 & 0 & 0 & 0 & 5 & 0 & 13 \\
\hline 2011 & 0 & 0 & 1 & 0 & 0 & 0 & 0 & 0 & 8 & 2 & 11 \\
\hline $\begin{array}{l}\text { Total } \mathbf{p} / \\
\text { Evento }\end{array}$ & 3 & 1 & 22 & 30 & 2 & 6 & 1 & 1 & 29 & 11 & - \\
\hline
\end{tabular}

Tabela 4 - Ano x Linhas de Pesquisa x Número de Artigos

\begin{tabular}{|c|c|c|c|c|c|c|c|c|c|c|c|c|c|c|}
\hline \multirow{2}{*}{ 窇忽 } & \multicolumn{2}{|c|}{2005} & \multicolumn{2}{|c|}{2006} & \multicolumn{2}{|c|}{2007} & \multicolumn{2}{|c|}{2008} & \multicolumn{2}{|c|}{2009} & \multicolumn{2}{|c|}{2010} & \multicolumn{2}{|c|}{2011} \\
\hline & $\begin{array}{c}\text { N. de } \\
\text { Artigos }\end{array}$ & $\%$ & $\begin{array}{l}\text { N. de } \\
\text { Artigos }\end{array}$ & $\%$ & $\begin{array}{c}\text { N. de } \\
\text { Artigos }\end{array}$ & $\%$ & $\begin{array}{c}\text { N. de } \\
\text { Artigos }\end{array}$ & $\%$ & $\begin{array}{c}\text { N. de } \\
\text { Artigos }\end{array}$ & $\%$ & $\begin{array}{l}\text { N. de } \\
\text { Artigos }\end{array}$ & $\%$ & $\begin{array}{c}\text { N. de } \\
\text { Artigos }\end{array}$ & $\%$ \\
\hline 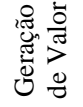 & 3,0 & 10,3 & 3,0 & 10,0 & 3,0 & 33,3 & 6,0 & 26,1 & 6,0 & $\begin{array}{c}26, \\
1\end{array}$ & 9,0 & 52,9 & 7,0 & 35,0 \\
\hline 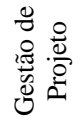 & 8,0 & 27,6 & 6,0 & 20,0 & 1,0 & 11,1 & 3,0 & 13,0 & 5,0 & $\begin{array}{c}21, \\
7\end{array}$ & 2,0 & 11,8 & 6,0 & 30,0 \\
\hline 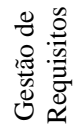 & 11,0 & 37,9 & 13,0 & 43,3 & 2,0 & 22,2 & 9,0 & 39,1 & 8,0 & $\begin{array}{c}34, \\
8\end{array}$ & 3,0 & 17,6 & 6,0 & 30,0 \\
\hline \& & 7,0 & 24,1 & 8,0 & 26,7 & 3,0 & 33,3 & 5,0 & 21,7 & 4,0 & $\begin{array}{c}17, \\
4\end{array}$ & 3,0 & 17,6 & 1,0 & 5,0 \\
\hline$\stackrel{5}{\frac{\pi}{6}}$ & 29 & 100 & 30 & 100 & 9 & 100 & 23 & 100 & 23 & 100 & 17 & 100 & 20 & 100 \\
\hline
\end{tabular}




\section{Análise das publicações por universidade}

Na Tabela 5, tem-se a apresentação da frequência de artigos publicados por universidade. Vê-se que dos 124 artigos levantados 32 tiveram a participação da UFRGS. O fato de poder haver mais de uma universidade vinculada a um artigo faz com que a soma total da frequência seja 149, e não 124, como seria numa relação unívoca. A diferença entre a Universidade com maior número de publicações e a segunda com o maior número de publicações é significativa (18 artigos). Observa-se a predominância de publicações de universidades nacionais nos eventos analisados, pois somente 2 universidades internacionais tiveram destaque. $\mathrm{O}$ fato de a maior parte desses eventos ser nacional pode ter tendenciado tal resultado.

A Tabela 6 apresenta um resumo da relação entre as universidades e as linhas de pesquisas. Das 44 universidades analisadas 20 são internacionais e apresentaram como principal linha de pesquisa Geração de Valor. Contudo, somente 2 apresentaram um número de publicações superior a 3 e, por isso, somente elas estão visíveis na Tabela 6. Já no contexto nacional, Gestão de Requisitos foi a linha de pesquisa mais explorada.

Como mencionado na seção 3, alguns artigos foram enquadrados em mais de uma linha de pesquisa, o que explica a divergência dos somatórios do número de artigos da Tabela 1 e do número de abordagens da Tabela 6 (124 e 173 respectivamente).

Figura 5 - Universidades $x$ N. de Artigos $x \%$ da amostra

\begin{tabular}{c|c|c}
\hline Universidades & N. de Artigos & \% da Amostra \\
\hline UFRGS & 32 & 21,5 \\
UFC & 14 & 9,4 \\
UFSC & 9 & 6,0 \\
UFPel & 8 & 5,4 \\
University of Salford - Reino Unido & 7 & 4,7 \\
USP & 6 & 4,0 \\
UFRJ & 5 & 3,4 \\
Unicamp & 5 & 3,4 \\
Luleå University of Technology - Suécia & 4 & 2,7 \\
Ufop & 4 & 2,7 \\
UFPE & 4 & 2,7 \\
Somatório das demais universidades & 51 & 34,2 \\
com número de artigos <4 & $\mathbf{1 4 9}$ & $\mathbf{1 0 0 , 0}$ \\
\hline TOTAL &
\end{tabular}

Tabela 6 - Universidades x Linhas de Pesquisa x Número de Abordagens das Linhas de Pesquisa

\begin{tabular}{c|c|c|c|c|c}
\hline Universidades & $\begin{array}{c}\text { Geração de } \\
\text { Valor }\end{array}$ & $\begin{array}{c}\text { Gestão de } \\
\text { Projeto }\end{array}$ & $\begin{array}{c}\text { Gestão de } \\
\text { Requisitos }\end{array}$ & APO & Total \\
\hline UFRGS & 7 & 6 & 20 & 6 & 39 \\
UFC & 5 & 8 & 5 & 2 & 20 \\
UFPel & 4 & 1 & 4 & 4 & 9 \\
University of Salford - Reino Unido & 3 & 2 & & 9 \\
UFSC & & 2 & 5 & 2 & 9 \\
USP & & 2 & & 4 & 6 \\
UFPE & & & 4 & 2 & 6 \\
UFRJ & 3 & 1 & 1 & 2 & 5 \\
Unicamp & 4 & & 1 & & 5 \\
UFMG & 1 & 3 & & 2 & 5 \\
Cefet-PB & 1 & 2 & 1 & & 4 \\
UEL & 17 & 6 & 14 & 10 & 47 \\
\hline Luleå University of Technology & $\mathbf{4 2}$ & $\mathbf{3 5}$ & $\mathbf{6 0}$ & $\mathbf{3 6}$ & $\mathbf{1 7 3}$ \\
\hline
\end{tabular}




\section{Análise das publicações por autores}

A Tabela 7 apresenta o número de artigos publicados de acordo com a titulação dos autores, contabilizando 370 autores/coautores. Através dela é possível observar suas qualificações técnicocientíficas.

Como o esperado, nota-se uma frequência superior de doutores nas publicações $(24,4 \%)$. Contudo, o número de titulações não indicadas apresentou-se com maior porcentagem $(43,2 \%)$, caracterizandose como uma oportunidade de melhoria no processo de produção científica, pois é interessante a exposição do título dos autores como indício do nível de experiência deles. Nesse grupo, é possível que estejam incluídos um número significativo de outras titulações, o que poderia vir a alterar a composição da Tabela 7.

Os doutores e os estudantes de doutorado são os únicos grupos acadêmicos presentes em todos os anos analisados, como observado na Tabela 8. O único grupo que apresentou crescimento durante o período estudado foi o de estudantes de doutorado.

O número de autores sem titulação indicada é bastante significativo, chegando a representar mais de $50 \%$ nos anos de 2007, 2010 e 2011, como se nota na Tabela 8 . Ao se analisarem as frequências relacionadas aos estudantes, percebe-se maior atividade dos estudantes de mestrado nos anos de 2008 e 2009, o que poderia indicar uma tendência da época em relação ao foco do requisito do cliente entre os mestrandos brasileiros.

Tabela 7 - Titulação x Número de Autorias x \% da Amostra

\begin{tabular}{c|c|c}
\hline Titulação Acadêmica & Autoria de Artigos & \% da Amostra \\
\hline Doutor & 90 & 24,4 \\
Estudante de Doutorado & 21 & 5,7 \\
Mestre & 27 & 7,3 \\
Estudante de Mestrado & 29 & 7,8 \\
Graduado & 19 & 5,1 \\
Estudante de Graduação & 19 & 5,1 \\
Especialista & 4 & 1,1 \\
MBA & 1 & 0,3 \\
Não Indicado & 160 & 43,2 \\
\hline TOTAL & $\mathbf{3 7 0}$ & $\mathbf{1 0 0 , 0}$
\end{tabular}

Tabela 8 - Titulação x Ano x Número de Autores

\begin{tabular}{|c|c|c|c|c|c|c|c|c|c|c|c|}
\hline & 莺 & $\begin{array}{l}\dot{\vec{\theta}} \\
\dot{\Xi}\end{array}$ & 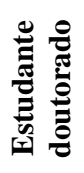 & 离 & 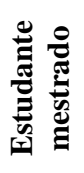 & 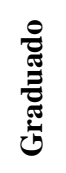 & 苞 & 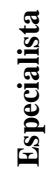 & 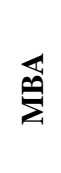 & 疍 & हే \\
\hline \multirow{2}{*}{2011} & $\%$ & 7,1 & 14,3 & 14,3 & 0,0 & 0,0 & 2,4 & 0,0 & 0,0 & 61,9 & 100,0 \\
\hline & Número & 3 & 6 & 6 & 0 & 0 & 1 & 0 & 0 & 26 & 42 \\
\hline \multirow{2}{*}{2010} & $\%$ & 14,0 & 7,0 & 4,7 & 2,3 & 4,7 & 0,0 & 0,0 & 0,0 & 67,4 & 100,0 \\
\hline & Número & 6 & 3 & 2 & 1 & 2 & 0 & 0 & 0 & 29 & 43 \\
\hline \multirow{2}{*}{2009} & $\%$ & 32,3 & 4,8 & 0,0 & 25,8 & 3,2 & 8,1 & 0,0 & 0,0 & 25,8 & 100,0 \\
\hline & Número & 20 & 3 & 0 & 16 & 2 & 5 & 50 & 0 & 16 & 62 \\
\hline \multirow{2}{*}{2008} & $\%$ & 40,7 & 5,1 & 6,8 & 15,3 & 1,7 & 6,8 & 0,0 & 0,0 & 23,7 & 100,0 \\
\hline & Número & 24 & 3 & 4 & 9 & 1 & 4 & 0 & 0 & 14 & 59 \\
\hline \multirow{2}{*}{2007} & $\%$ & 4,5 & 9,1 & 4,5 & 0,0 & 9,1 & 0,0 & 0,0 & 0,0 & 72,7 & 100,0 \\
\hline & Número & 1 & 2 & 1 & 0 & 2 & 0 & 0 & 0 & 16 & 22 \\
\hline \multirow{2}{*}{2006} & $\%$ & 25,7 & 2,7 & 8,1 & 4,1 & 5,4 & 5,4 & 1,4 & 0,0 & 47,3 & 100,0 \\
\hline & Número & 19 & 2 & 6 & 3 & 4 & 4 & 1 & 0 & 35 & 74 \\
\hline \multirow{2}{*}{2005} & $\%$ & 25 & 2,9 & 11,8 & 0,0 & 11,8 & 7,4 & 4,4 & 1,5 & 35,3 & 100,0 \\
\hline & Número & 17 & 2 & 8 & 0 & 8 & 5 & 3 & 1 & 24 & 68 \\
\hline
\end{tabular}

102 Vasconcelos, I. A. de; Soares, M. F.; Heineck, L. F. M.; Barros Neto, J. de P. 
Dos 124 artigos analisados foram gerados 697 autorias/coautorias. Na Tabela 9, contudo, são representados somente autores/coautores com considerável assiduidade (3 ou mais publicações). Observa-se que universidades do Sul do país têm um número maior de pesquisadores e publicações na temática analisada, perfazendo em torno de $13,8 \%$ do total de publicações e um grupo de 16 pesquisadores. As regiões Sudeste e Nordeste também aparecem com destaque (respectivamente $1,7 \%$ e $3,9 \%$ das autorias, com 4 pesquisadores cada). Em âmbito internacional, é possível observar a existência de grupos no Reino Unido e Suécia que tratam da linha de pesquisa abordada neste artigo.

\section{Análise das publicações de acordo com a Estratégia de Pesquisa}

A Tabela 10 apresenta que $43,55 \%$ da amostra utilizou estudo de caso como metodologia, muitos deles através de uma APO, sendo esse conjunto de métodos e técnicas também utilizado em alguns poucos casos de levantamento, o que fez com que fosse determinado pelos autores deste artigo que, caso o autor não indicasse sua metodologia claramente, a estratégia de pesquisa seria caracterizada como "não indicada", devido às divergências nas classificações. Por isso, esse grupo representou $17,74 \%$ da amostra, sendo o terceiro grupo mais representativo.

Tabela 9 - Número de Autores Assíduos x Universidades x Número de Publicações

\begin{tabular}{|c|c|c|c|c|c|}
\hline \multicolumn{6}{|c|}{ Universidades Nacionais } \\
\hline Região & $\begin{array}{l}\text { Número de Autores } \\
\text { Assíduos (mais de } 3 \\
\text { publicações) }\end{array}$ & Universidade & $\begin{array}{l}\text { Publicações } \\
\text { (Autor) }\end{array}$ & $\begin{array}{l}\text { Publicações } \\
\text { (Coautor) }\end{array}$ & Total \\
\hline Sul & 10 & UFRGS & 18 & 45 & 63 \\
\hline Sul & 4 & UFPEL & 12 & 14 & 26 \\
\hline Sul & 2 & UFSC & 2 & 5 & 7 \\
\hline Sudeste & 2 & UFOP & 3 & 3 & 6 \\
\hline Sudeste & 1 & UNICAMP & 1 & 2 & 3 \\
\hline Sudeste & 1 & UFMG & 2 & 1 & 3 \\
\hline Nordeste & 3 & UFC & 6 & 18 & 24 \\
\hline Nordeste & 1 & UECE & 1 & 2 & 3 \\
\hline \multicolumn{6}{|c|}{$\begin{array}{cc}\text { Universidades Internacionais } \\
\end{array}$} \\
\hline Região & $\begin{array}{c}\text { Número de Autores } \\
\text { Assíduos (mais de } 3 \\
\text { publicações) }\end{array}$ & Universidade & $\begin{array}{l}\text { Publicações } \\
\text { (Autor) }\end{array}$ & $\begin{array}{l}\text { Publicações } \\
\text { (Coautor) }\end{array}$ & Total \\
\hline Reino Unido & 2 & University of Salford & 2 & 5 & 4 \\
\hline Reino Unido & 2 & $\begin{array}{l}\text { Loughborough } \\
\text { University }\end{array}$ & 3 & 3 & 3 \\
\hline Suécia & 1 & $\begin{array}{c}\text { Luleå University of } \\
\text { Technology }\end{array}$ & 1 & 2 & 3 \\
\hline \multicolumn{6}{|c|}{ Sem Origem Informada } \\
\hline Região & $\begin{array}{l}\text { Número de Autores } \\
\text { Assíduos (mais de } 3 \\
\text { publicações) }\end{array}$ & Universidade & $\begin{array}{l}\text { Publicações } \\
\text { (Autor) }\end{array}$ & $\begin{array}{l}\text { Publicações } \\
\text { (Coautor) }\end{array}$ & Total \\
\hline- & 2 & - & 2 & 4 & 3 \\
\hline
\end{tabular}

Tabela 10 - Estratégia de Pesquisa x Número de Artigos x \% da Amostra

\begin{tabular}{c|c|c}
\hline Estratégia de Pesquisa & N. de Artigos & \% da Amostra \\
\hline Estudo Teórico & 8 & 6,45 \\
Estudo de Caso & 54 & 43,55 \\
Aplicação de Metodologia & 7 & 5,65 \\
Levantamento & 28 & 22,58 \\
Pesquisa-ação & 2 & 1,61 \\
Pesquisa Construtiva & 3 & 2,42 \\
Não Indicado & 22 & 17,74 \\
\hline TOTAL & $\mathbf{1 2 4}$ & $\mathbf{1 0 0}$ \\
\hline
\end{tabular}


Aprofundando este estudo, construiu-se a Tabela 11, que representa uma análise da quantidade de artigos por estratégia ao longo dos anos. O Estudo de Caso e Levantamento foram os únicos grupos que estiveram presentes em todos os sete anos analisados.

Na Tabela 12, aborda-se ainda a estratégia de pesquisa, analisando-se seu detalhamento.

Tabela 11 - Estratégia de Pesquisa x Ano x Número de Artigos

\begin{tabular}{|c|c|c|c|c|c|c|c|c|c|c|c|c|c|c|}
\hline \multirow{2}{*}{ 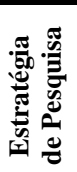 } & \multicolumn{2}{|c|}{2005} & \multicolumn{2}{|c|}{2006} & \multicolumn{2}{|c|}{2007} & \multicolumn{2}{|c|}{2008} & \multicolumn{2}{|c|}{2009} & \multicolumn{2}{|c|}{2010} & \multicolumn{2}{|c|}{2011} \\
\hline & $\begin{array}{l}\text { N. de } \\
\text { Artigos }\end{array}$ & $\%$ & $\begin{array}{l}\text { N. de } \\
\text { Artigos }\end{array}$ & $\%$ & $\begin{array}{c}\text { N. de } \\
\text { Artigos }\end{array}$ & $\%$ & $\begin{array}{l}\text { N. de } \\
\text { Artigos }\end{array}$ & $\%$ & $\begin{array}{c}\text { N. de } \\
\text { Artigos }\end{array}$ & $\%$ & $\begin{array}{c}\text { N. de } \\
\text { Artigos }\end{array}$ & $\%$ & $\begin{array}{l}\text { N. de } \\
\text { Artigos }\end{array}$ & $\%$ \\
\hline 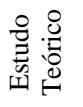 & 0 & 0,0 & 2 & 7,7 & 0 & 0,0 & 2 & 9,1 & 2 & 10,5 & 1 & 7,7 & 1 & 7,1 \\
\hline 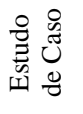 & 11 & 45,8 & 12 & 46,2 & 3 & 50,0 & 12 & 54,5 & 7 & 36,8 & 6 & 46,2 & 3 & 21,4 \\
\hline 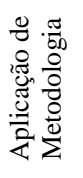 & 1 & 4,2 & 0 & 0,0 & 0 & 0,0 & 0 & 0,0 & 1 & 5,3 & 2 & 15,4 & 3 & 21,4 \\
\hline 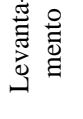 & 6 & 25,0 & 5 & 19,2 & 1 & 16,7 & 5 & 22,7 & 6 & 31,6 & 2 & 15,4 & 3 & 21,4 \\
\hline 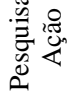 & 1 & 4,2 & 0 & 0,0 & 0 & 0,0 & 0 & 0,0 & 0 & 0,0 & 0 & 0,0 & 1 & 7,1 \\
\hline 胥 & 0 & 0,0 & 0 & 0,0 & 0 & 0,0 & 0 & 0,0 & 0 & 0,0 & 1 & 7,7 & 2 & 14,3 \\
\hline 胥 & 5 & 20,8 & 7 & 26,9 & 2 & 33,3 & 3 & 13,6 & 3 & 15,8 & 1 & 7,7 & 1 & 7,1 \\
\hline ) & 24 & 100 & 26 & 100 & 6 & 100 & 22 & 100 & 19 & 100 & 13 & 100,0 & 14 & 100,0 \\
\hline
\end{tabular}

Tabela 12 - Estratégia de Pesquisa x Detalhamento de Método x \% da Amostra

\begin{tabular}{c|c|c|c|c}
\hline Estratégia de Pesquisa & \multicolumn{3}{|c|}{ Detalhamento } & \multicolumn{2}{c}{ \% da Amostra } \\
& Sim & Não & Sim & Não \\
\hline Estudo Teórico & 3 & 5 & 3,33 & 14,71 \\
Estudo de Caso & 46 & 8 & 51,11 & 23,53 \\
Aplicação de Metodologia & 6 & 1 & 6,67 & 2,94 \\
Levantamento & 23 & 5 & 25,56 & 14,71 \\
Pesquisa-Ação & 2 & 0 & 2,22 & 0,00 \\
Pesquisa Construtiva & 3 & 0 & 3,33 & 0,00 \\
Não Indicado & 7 & 15 & 7,78 & 44,12 \\
\hline TOTAL ARTIGOS & $\mathbf{9 0}$ & $\mathbf{3 4}$ & $\mathbf{7 2 , 5 8}$ & $\mathbf{2 7 , 4 2}$ \\
\hline
\end{tabular}


Como se optou por atribuir "não indicado" a todo artigo que não classificasse diretamente sua metodologia, 7 deles apresentaram detalhamento. Apesar de a maioria dos artigos ter explicado sua metodologia, o número de artigos sem detalhamento ainda é muito alto, 27,42\%, principalmente nos artigos internacionais.

\section{Análise das publicações por bibliografia}

Foram levantadas 2.038 bibliografias, sendo destas 1.454 diferentes. Entre elas, os Livros, os Artigos de Periódicos e os Artigos de Eventos são os três tipos de maior representatividade, pois correspondem a $79,51 \%$ das bibliografias encontradas, seguidos pelas Teses e Dissertações $(9,77 \%)$. Nota-se que uma grande parte das referências são artigos, já que elas constituem $49,45 \%$ da amostra. Esses dados constam na Tabela 13.

A Tabela 14 apresenta as 9 bibliografias mais citadas, sendo 2 delas do mesmo autor, o que ilustra que o autor da bibliografia mais utilizada não necessariamente é o mais mencionado.

Entre as mais referenciadas, há duas sobre APO, conjunto de métodos e técnicas bastante utilizado na metodologia, sendo por isso uma das linhas de pesquisas adotadas neste artigo para a caracterização.

Koskela e Miron, dois autores que apresentaram um número elevado de publicações no período analisado, também são autores evidenciados entre as bibliografias mais utilizadas, o que pode ser constatado na Tabela 14. Ratifica-se que a Tabela 14 não apresenta os autores mais citados pelos artigos, mas as bibliografias mais utilizadas.

\section{Conclusão}

O presente artigo apresentou uma caracterização da produção científica sobre requisitos do cliente do mercado da construção civil entre 2005 e 2011, com foco nos eventos SIBRAGEC, ENTAC e IGLC, e na Revista Ambiente Construído, atingindo o objetivo a que se propôs.

A partir do projeto de Miron (2005), houve um pico de publicações sobre requisitos dos clientes, que gradativamente diminuiu ao longo dos anos e eventos analisados, consolidando um grupo reduzido de pesquisadores, concentrados nas universidades UFRGS e UFC, que sozinhas representaram 30,9\% das publicações analisadas.

Como tendência de produção científica relacionada ao tema, destaca-se a linha de pesquisa de Gestão de Requisitos, por apresentar maior número de trabalhos tratando dessa vertente $(34,44 \%$ da amostra). Outras linhas também se apresentaram como alvo de encaminhamentos de pesquisa, tais como Geração de Valor, Gestão de Projetos e APO (de $20 \%$ a $25 \%$ da amostra cada).

Com relação ao método de pesquisa, percebeu-se uma oportunidade de melhoria conceitual e estrutural. A primeira, por alguns autores terem classificado metodologias iguais de formas diferentes; e a segunda pelo alto índice $(17,74 \%)$ de publicações sem a indicação de qual metodologia foi utilizada na pesquisa e pelo elevado número $(27,42 \%)$ de artigos sem o detalhamento dela. É importante ressaltar que, à exceção do periódico da revista Ambiente Construído, os dados analisados são provenientes de congressos, o que limitou a análise do rigor científico das publicações, pois nesses eventos os artigos costumam ser apresentados de forma resumida e, geralmente, se concentram mais nos resultados obtidos do que no procedimento de pesquisa empregado. As estratégias de pesquisa que mais se destacaram foram Estudo de Caso $(43,55 \%)$ e Levantamento (22,58\%), ressaltando que a Pesquisa Construtiva apareceu somente nos últimos anos, o que indica uma possível nova vertente no uso dessa estratégia.

Tabela 13 - Tipo de Bibliografia x Número de Citações x \% Amostra

\begin{tabular}{c|c|c}
\hline Tipo de Bibliografia & N. de Citações & \% da Amostra \\
\hline Livros & 437 & $30,06 \%$ \\
Artigos de Evento & 298 & $20,50 \%$ \\
Artigos de Periódicos & 421 & $28,95 \%$ \\
Teses ou Dissertações & 142 & $9,77 \%$ \\
Relatórios de Pesquisa & 30 & $2,06 \%$ \\
Normas Técnicas & 26 & $1,79 \%$ \\
Legislações & 10 & $0,69 \%$ \\
Boletins Técnicos & 16 & $1,10 \%$ \\
Outros & 74 & $5,09 \%$ \\
\hline TOTAL & $\mathbf{1 . 4 5 4}$ & $\mathbf{1 0 0}$ \\
\hline
\end{tabular}


Tabela 14 - Referências mais Citadas x Tipo de Referência x Número de Citações

\begin{tabular}{|c|c|c|}
\hline Referências mais Citadas & Tipo & N. de Citações \\
\hline $\begin{array}{l}\text { ORNSTEIN, S. W. Avaliação pós-ocupação (APO) do ambiente construído. } \\
\text { Colab. Marcelo Roméro. São Paulo. Editoras Studio Nobel e da Universidade de } \\
\text { São Paulo, } 1992\end{array}$ & Livro & 17 \\
\hline $\begin{array}{l}\text { KOSKELA, L. An exploration towards a production theory and its application to } \\
\text { construction. } 296 \mathrm{f} \text {. Thesis. (Doctor of Technology). Technical Research Centre of } \\
\text { Finland - VTT. Helsinki, 2000. }\end{array}$ & $\begin{array}{c}\text { Tese ou } \\
\text { Dissertação }\end{array}$ & 15 \\
\hline $\begin{array}{l}\text { CAIXA ECONÔMICA FEDERAL. Programa de Arrendamento Residencial. } \\
\text { Brasília, 2003. Disponível em: } \\
\text { <https://webp.caixa.gov.br/urbanizacao/Publicacao/Texto/programa/PAR.htm>. } \\
\text { Acesso em: } 20 \text { dez } 2003 .\end{array}$ & Outro & 11 \\
\hline $\begin{array}{l}\text { KAMARA, J. M.; ANUMBA, C. J.; EVBUOMWAN, N. F. Establishing and } \\
\text { processing client requirements: a key aspect of concurrent engineering in } \\
\text { construction. Engineering, Construction and Architectural Management, v. 7, n. 1, } \\
\text { p. 15- 28, Mar. 2000. }\end{array}$ & $\begin{array}{l}\text { Artigo de } \\
\text { periódico }\end{array}$ & 9 \\
\hline $\begin{array}{l}\text { MIRON, L. Proposta de modelo para o gerenciamento de requisitos do cliente em } \\
\text { empreendimentos habitacionais de interesse social. 2004. } 21 \text { f. Projeto de Pesquisa } \\
\text { (Doutorado em Engenharia Civil) - Escola de Engenharia, Universidade Federal } \\
\text { do Rio Grande do Sul, Porto Alegre, 2004. }\end{array}$ & $\begin{array}{c}\text { Relatório de } \\
\text { Pesquisa }\end{array}$ & 8 \\
\hline $\begin{array}{l}\text { ORNSTEIN, S.; BRUNA, G.; ROMÉRO, M. Ambiente construído \& } \\
\text { comportamento: avaliação pós-ocupação e a qualidade ambiental. São Paulo. } \\
\text { Editorial Studio Nobel, Fundação para a Pesquisa Ambiental e Faculdade de } \\
\text { Arquitetura e Urbanismo da USP, } 1995 .\end{array}$ & Livro & 8 \\
\hline $\begin{array}{l}\text { EMMIT, S.; SANDER, D.; CHRISTOFFERSEN, A. K. The value universe: } \\
\text { defining a value based approach to lean construction. Proc., 13th Annual } \\
\text { Conference on Lean Construction, IGLC, Sydney, Australia, 2005. p. 57-64 . }\end{array}$ & $\begin{array}{l}\text { Artigo de } \\
\text { Evento }\end{array}$ & 8 \\
\hline $\begin{array}{l}\text { SALIBA, M.; FISHER, C. Managing customer value: a framework allows } \\
\text { organisations to achieve and sustain competitive advantage. Quality Progress, v. } \\
33 \text {, n. 6, p. 63-69, Jun. } 2000 \text {. }\end{array}$ & $\begin{array}{l}\text { Artigo de } \\
\text { Periódico }\end{array}$ & 7 \\
\hline \multirow[t]{2}{*}{$\begin{array}{l}\text { KAMARA, J. M.; ANUMBA, C. J.; EVBUOMWAN, N. F. O. Client } \\
\text { requirements processing in construction: a new approach using QFD. Journal of } \\
\text { Architectural Engineering, v. 5, n. 1, p. 8-15, Mar. } 1999 .\end{array}$} & $\begin{array}{l}\text { Artigo de } \\
\text { Periódico }\end{array}$ & 7 \\
\hline & Diverso & $<7$ \\
\hline
\end{tabular}

A análise das referências bibliográficas utilizadas pelos artigos indica um conservadorismo com a fonte de pesquisa, que se fortalece diante dos tipos de literatura mais utilizados: Livros $(30,06 \%)$ e Artigos de Periódicos $(28,95 \%)$. Com relação às referências mais citadas, destacam-se os autores Ornstein, Koskela, Kamara, Miron, Emmit e Saliba, cujos trabalhos enquadram-se nas linhas de pesquisa estabelecidas neste estudo.

Se seguisse as tendências apresentadas pelos dados analisados, um novo artigo sobre o tema estudado abordaria como linha de pesquisa Geração de Requisitos do Cliente, seria desenvolvido nas regiões Sul e/ou Nordeste do país, com orientação e participação de doutores, apresentando como metodologia o estudo de caso e baseando-se principalmente em livros e artigos de periódicos. Vale ressaltar que esse perfil não necessariamente representa a melhor apresentação de artigo a ser desenvolvido para esse tema, mas um artigo com as principais tendências constatadas neste trabalho.
Devido à elevada quantidade de trabalhos acadêmicos produzidos anualmente, que fornecem um grande número de dados, bibliométricos ou não, sugere-se a automatização da coleta e processamento de dados. É recomendada a inclusão de outros periódicos, como Gestão \& Tecnologia de Projetos e Facilities e Architectural Engineering and Design Management, assim como a expansão dos dados a serem analisados, tais como idioma dos artigos e das referências utilizadas neles, estrutura lógica dos trabalhos, relevância e originalidade deles.

\section{Referências}

BAILETTI, A. J.; LITVA, P. F. Integrating Customer Requirements Into Product Designs. Journal of Production Innovation Management, v. 12, n. 1, p. 3-15, 1995. 
BALLARD, G. Lean Project Delivery System. White Paper-8 (Revision 1), Lean Construction Institute, Ketchum, Id. 23 sep. 2000. Disponível em: <http://www.leanconstruction.org/pdf/WP8LPDS.pdf $>$. Acesso em 05 mar. 2013.

BARRETT, P. S.; HUDSON, J.; STANLEY, C. Good Practice in Briefing: the Limits of Rationality. Automation in Construction, v. 8, n. 6, p. 633-642, 1999.

BRUCE, M.; COOPER, R. Creative Product Design: a practical guide to requirements capture management. Chichester: John Wiley \& Sons, 2000.

FONSECA, E. N. (Org). Bibliometria: teoria e prática. São Paulo: Cultrix, Ed. da USP, 1986.

FORMOSO, C. T. Lean Construction: princípios básicos e exemplos. Pini, 10 out. 2002. Disponível em:

<www.piniweb.com.br/construcao/noticias/leanconstruction-principios-basicos-e-exemplos80714-1.asp>. Acesso em: 05 mar.2013.

FORMOSO, C. T.; LEITE, F. L.; MIRON, L. I. G. Client Requirements Management in Social Housing: case study of the residential leasing program in Brazil. Journal of Construction in Developing Countries, v. 16, n. 2, p. 47-67, 2011.

GUEDES, V.; BORSCHIVER, S. Bibliometria: uma ferramenta estatística para a gestão da informação e do conhecimento, em sistemas de informação, de comunicação e de avaliação científica e tecnológica. In: ENCONTRO NACIONAL DE CIÊNCIA DA INFORMAÇÃO, 6., Salvador, 2005. Anais... Salvador: ICI/UFBA, 2005.

HUOVILA, P.; SERÉN, K. J. Customer-Oriented Design for Construction Projects. Journal of Engineering Design, v. 9, n. 3, p. 225-238, 1998.

KAMARA, J. M.; ANUMBA, C. J.; EVBUOMWAN, N. F. O. Capturing Client Requirements in Construction Projects. Reston: Thomas Telford, 2002.

KOSKELA, L. An Exploration Towards a Production Theory and Its Application to Construction. Espoo 2000, Technical Research Centre of Finland, VTT Publications 408, 296p, 2000.

KRZYZANOWSKI, R.; FERREIRA, M. C. Avaliação de Periódicos Científicos e Técnicos Brasileiros. Ciência da Informação, Brasília, v. 27, n. 2, p. 165-175, maio/ago. 1998.
MACIAS-CHAPULA, C. A. O Papel da Informetria e da Cientometria e Sua Perspectiva Nacional e Internacional. Ciência da Informação, Brasília, v. 27, n. 2, p. 134-140, maio/ago. 1998.

MIRON, L. I. G. Proposta de Modelo Para o Gerenciamento de Requisitos do Cliente em Empreendimentos Habitacionais de Interesse Social. 21 f. Porto Alegre, 2004. Tese (Doutorado em Engenharia Civil) - Escola de Engenharia, Universidade Federal do Rio Grande do Sul, Porto Alegre, 2005.

MIRON, L. I. G.; FORMOSO, C. T. Client Requirements Management. In: ANNUAL CONFERENCE OF THE INTERNATIONAL GROUP FOR LEAN CONSTRUCTION, 11., Blacksburg, 2003. Proceedings... Blacksburg: Virginia Polytechnic Institute and State University, 2003.

MUGNANI, R.; JANNUZZI, P.; QUONIAM, L. Indicadores Bibliométricos da Produção Científica Brasileira: uma análise a partir da base Pascal.

Ciência da Informação, Brasília, v. 33, n. 2, p. 123-131, maio/ago. 2004.

PITHAN, D. N. et al. Caracterização da Produção Científica de Áreas de Conhecimento Específicas: aplicação à gestão e economia da construção.

Ambiente Construído, Porto Alegre, v. 5, n. 3, p. 7-18, jul./set. 2005.

ROUSEAU-HANS, F.; THIL, J. Veille et Informatique: des besoins aux solutions.

Technologies Internationales, Strasbourg, v. 39, p. 33-36, nov. 1997.

ROUSSEAU, R. Indicadores Bibliométricos e Econométricos Para a Avaliação de Instituições Científicas. Ciência da Informação, Brasília, v. 27, n. 2, p. 145-58, maio/ago. 1998.

SAES, G. S. Estudo Bibliométrico das Publicações em Economia da Saúde, no Brasil, 1989-1998. 2000. Dissertação (Mestrado em Saúde Pública) - Faculdade de Saúde Pública, Universidade de São Paulo, São Paulo, 2000.

SHEN, Q. et al. A Framework For Identification and Representation of Client Requirements in the Briefing Process. In: Construction Management and Economics, v. 22, p. 213-221, 2004.

TAGUE-SUTCLIFFE, J. An Introduction to Infometrics. Information Processing \& Management, Oxford, v. 28, n. 1, p. 1-3, 1992.

\section{Agradecimento}

À CAPES e ao PROPAG/REUNI, pelo apoio provido. 


\section{Revista Ambiente Construído}

Associação Nacional de Tecnologia do Ambiente Construído

Av. Osvaldo Aranha, $99-3^{\circ}$ andar, Centro

Porto Alegre - RS - Brasil

CEP 90035-190

Telefone: +55 (51) 3308-4084

Fax: +55 (51) 3308-4054

www.seer.ufrgs.br/ambienteconstruido

E-mail: ambienteconstruido@ufrgs.br

108 Vasconcelos, I. A. de; Soares, M. F.; Heineck, L. F. M.; Barros Neto, J. de P. 